den Einfluss der Bundespolitik direkt über die Einschätzung der Wähler zu beurteilen. In der individuellen Wahrnehmung der Bürger (direkte Einflussebene) spielt die Bundespolitik je nach Frageformulierung eine unterschiedliche Rolle. Zwar wird sie für die Wahlentscheidung als wichtig angesehen, im Vergleich mit der Landespolitik ist sie aber weniger bedeutsam. Unterschiede lassen sich insofern feststellen, als in den neuen Ländern der bundespolitische Einfluss von den Bürgern homogener wahrgenommen wird als im Westen. In den alten Bundesländern fällt auf, dass insbesondere in den Stadtstaaten die Bundespolitik eine besonders geringe Rolle spielt.

Die indirekte Messung der Relevanz der Bundespolitik bestätigt, wie wenig die Zufriedenheit mit der Bundesregierung die Stimmenabgabe bei Landtagswahlen beeinflusst. Im Vergleich zur Zufriedenheit mit der Landesregierung übt die Zufriedenheit mit der Bundesregierung lediglich dann einen Einfluss aus, wenn sich die von einem Bürger präferierte Partei im Bund in der Regierungsverantwortung befindet und auf Landesebene in der Opposition oder in einer Großen Koalition mitregiert. Hier kommt der oft zitierte „Rückenwind vom Bund“ zum Tragen. Erweitert man das Untersuchungsmodell allerdings um die Kandidatenorientierung in Form des bevorzugten Ministerpräsidenten, zeigt sich sowohl für CDU/CSU- als auch SPD-Wähler, dass die Zufriedenheit mit der Bundesregierung eine untergeordnete Rolle spielt. Noch deutlicher tritt dieses Ergebnis zu Tage, wenn zusätzlich die langfristig wirksame Parteibindung berücksichtigt wird, die den Haupterklärungsfaktor für die Wahlentscheidung der Bürger darstellt. Lediglich in Ausnahmesituationen können bundespolitische Themenorientierungen einen annähernd gleich starken Einfluss auf die Stimmenabgabe der Wähler ausüben wie die Zufriedenheit mit der Landesregierung oder der präferierte Kandidat für das Amt des Ministerpräsidenten.

Die Frage nach dem Bundeseinfluss scheint sich somit bei jeder Landtagswahl neu zu stellen. Allgemeine Tendenzen für die Wähler der beiden Volksparteien in den einzelnen Bundesländern und je nach Regierungs- oder Oppositionszugehörigkeit der bevorzugten Partei im Bundes- oder Landtag lassen sich nur schwer ausmachen.

\title{
Umfragedaten, Ereignisdaten und Makrodaten: Datenquellen für die Analyse der Landtagswahlen von 1960/62 bis 2004
}

\author{
Evelyn Brislinger
}

Die in diesem Heft der ZParl vorgestellten Analysen zum Einfluss der Bundespolitik auf Landtagswahlen basieren auf Umfrage-, Ereignis- und Makrodaten, die für die 16 Bundesländer zur Verfügung stehen und mehr als 40 Jahre umfassen. Das von der Deutschen Forschungsgemeinschaft (DFG) geförderte Forschungsprojekt ${ }^{1}$ hatte zum einen das Ziel,

1 Vgl. Oscar W. Gabriel / Everhard Holtmann / Wolfgang Jagodzinski, Einfluss der Bundespolitik auf Landtagswahlen - Eine Analyse des Wählerverhaltens auf Landesebene unter besonderer Berücksichtigung der Bundespolitik, Antrag an die DFG, November 2003. 
die Determinanten des Wahlverhaltens im Zeit- und Ländervergleich zu untersuchen und hierfür eine adäquate Datenbasis aufzubauen. Zum anderen sollte dieser Datenbestand nach Abschluss des Projektes schnell, ohne Mehraufwand und in einer benutzerfreundlichen Form für die wissenschaftliche Öffentlichkeit zur Verfügung stehen. Hierfür kooperieren das Institut für Sozialwissenschaften (SOWI I) der Universität Stuttgart, das Institut für Politikwissenschaft der Universität Halle-Wittenberg und das Zentralarchiv für Empirische Sozialforschung in Köln, um die enge Verbindung von Forschungsinteressen und Infrastrukturleistungen für die Forschung zu garantieren. Das Zentralarchiv als Teil der Gesellschaft Sozialwissenschaftlicher Infrastruktureinrichtungen (GESIS) konnte das Projekt durch die Bereitstellung eines nach internationalem Standard dokumentierten Datenbestandes unterstützen, für die Integration und Präsentation der Daten die erforderlichen Werkzeuge und Beratungsleistungen zur Verfügung stellen und wird die Ergebnisse über unterschiedliche Medien zugänglich machen.

\section{Zum Daten- und Informationsbestand}

Für den Zeitraum von 1962 bis 2004 stehen 105 Landtagswahlstudien in aufbereiteter Form für die sekundäranalytische Forschung zur Verfügung. Diese Studien (vgl. Tabelle 1) wurden als repräsentative Befragungen zumeist im Vorfeld der Landtagswahlen durchgeführt. Um vergleichende Analysen zu unterstützen, wurden die wahlsoziologisch relevanten Informationen der Einzelstudien am Institut für Sozialwissenschaften (SOWI I) der Universität Stuttgart in einen Gesamtdatensatz integriert. Die hierfür erforderliche Überprüfung der Vergleichbarkeit der Fragestellungen für die über 15.000 Variablen wurde durch den ZA CodebookExplorer unterstützt. Die Datenbank, im Europadatenlabor (ZA EUROLAB) für den Bestand der Bundestagsstudien ${ }^{2}$ entwickelt, wird in verschiedenen Projekten für die Präsentation komplexer Studienkollektionen eingesetzt. Sie ermöglicht dem interessierten Nutzer einen Überblick über den Studienbestand, die erfragten Themen sowie die verfügbaren Variablen.

Die Landtagswahlstudie aus Nordrhein-Westfalen, im Kontext der Kölner Wahlstudie 1962 entstanden, war eine der ersten empirischen Studien, die an das Zentralarchiv übergeben wurde. Es folgten ältere, die bis Mitte der 1970er Jahre von Forschergruppen an den Universitäten Mannheim und Köln sowie von der Gesellschaft für Markt-, Meinungs- und Sozialforschung (DIVO) in Frankfurt am Main durchgeführt wurden und der umfangreiche Bestand an jüngeren Umfragen, der vor allem durch die Forschungsgruppe Wahlen (FGW) kontinuierlich aufgebaut wird. Heute bilden die Landtagswahlstudien zusammen mit den Europa- und Bundestagswahlstudien, den Politbarometern, den European und East European Election Studies sowie den Wahlstudien zu den nationalen Parlamenten in Europa einen der stark nachgefragten Datenschwerpunkte des Archivs.

Für die Rekonstruierung der Kontexte, in denen die Landtagswahlen stattgefunden haben, wurden durch das Institut für Politikwissenschaft der Universität Halle-Wittenberg

2 Vgl. hierzu Wolfgang Zenk-Möltgen / Ekkehard Mochmann, Der Continuity Guide der deutschen Wahlforschung und der ZA CodebookExplorer, in: Markus Klein / Wolfgang Jagodzinski / Ekkehard Mochmann / Dieter Ohr (Hrsg.), 50 Jahre Empirische Wahlforschung in Deutschland, Opladen 2000, S. $596-614$. 


\begin{tabular}{|c|c|c|c|c|c|c|c|c|c|c|c|c|c|c|c|c|c|c|}
\hline 8 & 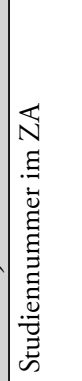 & 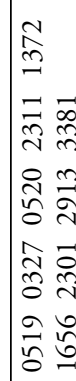 & 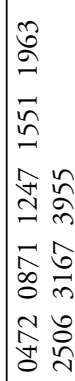 & 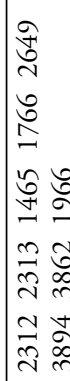 & 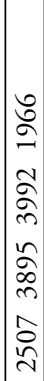 & 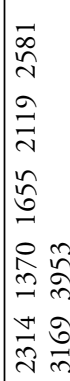 & 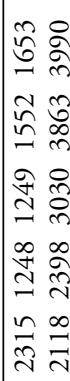 & 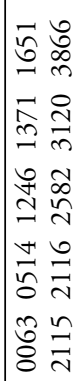 & 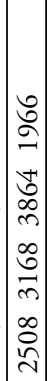 & 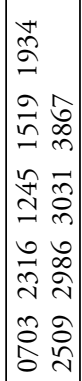 & 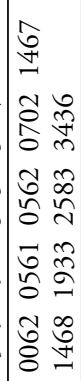 & 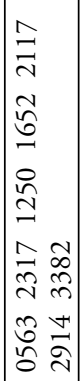 & 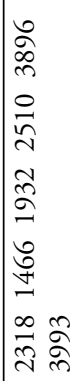 & 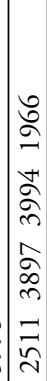 & 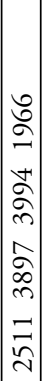 & 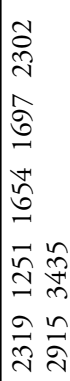 & 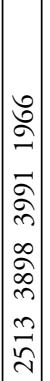 & 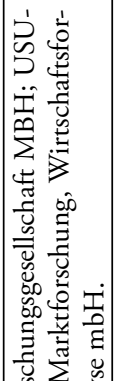 \\
\hline 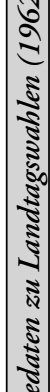 & 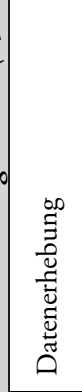 & 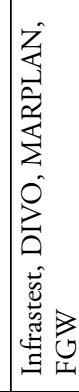 & 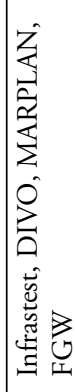 & 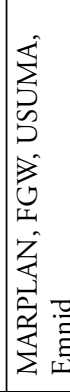 & 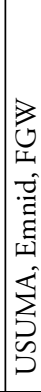 & 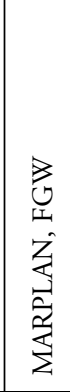 & 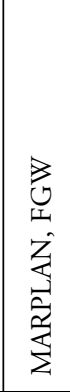 & 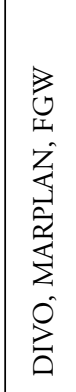 & 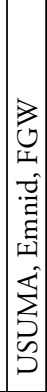 & 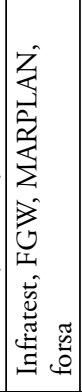 & 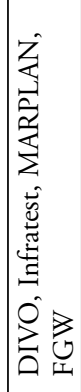 & 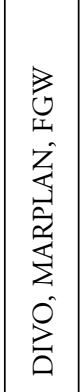 & 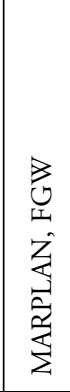 & 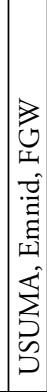 & 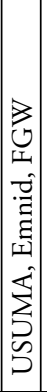 & 崩 & 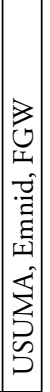 & 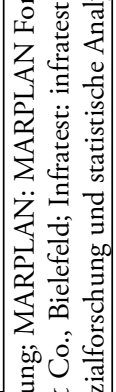 \\
\hline 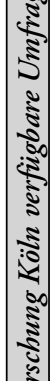 & 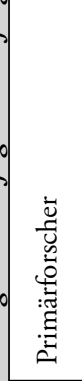 & 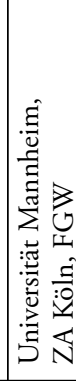 & 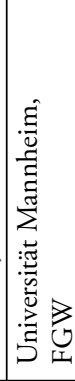 & 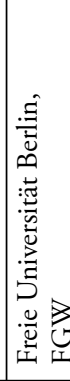 & 佡 & 总 & 离 & $\begin{array}{l}8 \\
0 \\
0 \\
\vdots \\
\vdots 0 \\
\vdots \\
\mathbb{N} \\
0 \\
0 \\
0\end{array}$ & $\begin{array}{l}8 \\
u \\
y\end{array}$ & 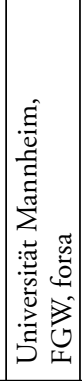 & 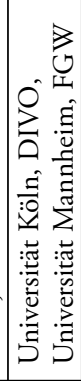 & 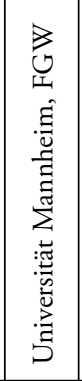 & 岁 & 署 & $\begin{array}{l}8 \\
0 \\
I\end{array}$ & 岁 & $\begin{array}{l}8 \\
0 \\
I\end{array}$ & 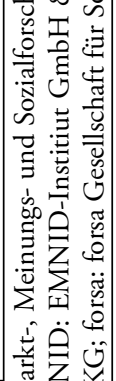 \\
\hline 过 & 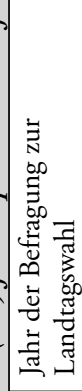 & 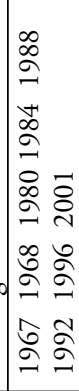 & 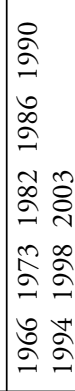 & 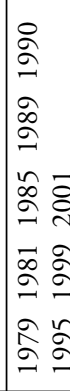 & 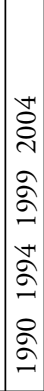 & 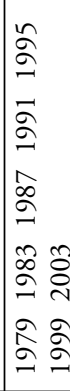 & 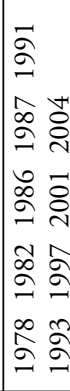 & 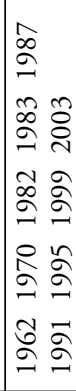 & 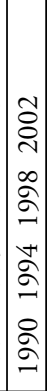 & 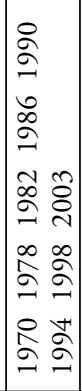 & 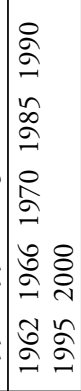 & 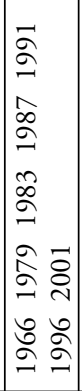 & 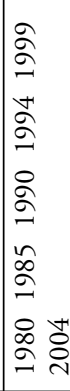 & 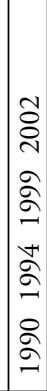 & 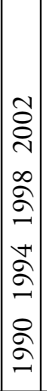 & 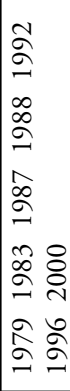 & 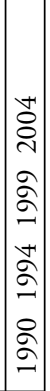 & 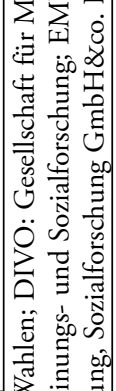 \\
\hline 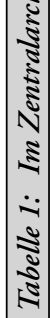 & 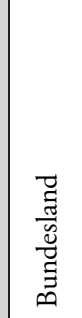 & 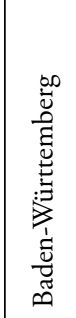 & 㲵 & 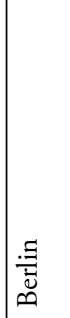 & 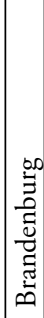 & $\begin{array}{l}\bar{\Xi} \\
\text { : } \\
\text { ص. }\end{array}$ & 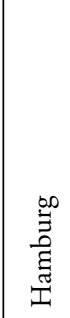 & 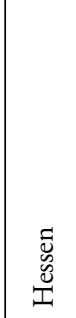 & 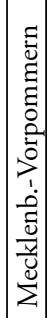 & 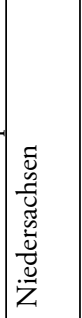 & 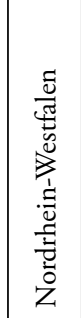 & 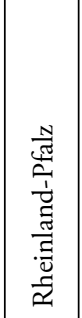 & 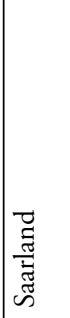 & 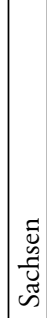 & 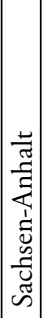 & 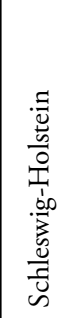 & $\mid$ & 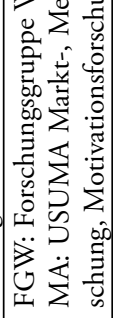 \\
\hline
\end{tabular}


zwei weitere Datensätze angelegt. Der Ergebnisdatenbestand gibt erstens Auskunft über politische Ereignisse sowie landes- und bundespolitische Stimmungslagen zum Zeitpunkt der Wahl (1960 bis 2004). Hierzu wurden eine Woche vor und nach dem jeweiligen Wahltermin die Frankfurter Allgemeine Zeitung und die Süddeutsche Zeitung inhaltsanalytisch nach wahlrelevanten Ereignissen untersucht. Die Daten basieren auf 4.328 analysierten Artikeln. Sie umfassen circa 1.800 Ereignisse, die unter anderem nach Art, Häufigkeit der Nennung sowie der erwarteten Wirkung auf die von dem Ereignis betroffenen Parteien kodiert wurden. Für Analysen auf Aggregatdatenebene stehen darüber hinaus die amtlichen Wahlergebnisse für die Bundes- und Landtagswahlen zur Verfügung (ebenfalls für 1960 bis 2004). Diese Daten wurden von den Statistischen Landesämtern auf der Ebene der administrativen Kreise (Landkreise, kreisfreie Städte sowie Bezirke in Berlin und Hamburg) bereitgestellt. Außerdem wurden Wirtschafts- und Sozialstrukturdaten wie Bruttoinlandsprodukt, Arbeitslosenquote, Anzahl der Sozialhilfeempfänger und sektorale Aufteilung der Erwerbstätigen als weitere Kontextmerkmale zur Beurteilung der wirtschaftlichen Lage in den Bundesländern erfasst.

Über diese Umfrage- und Kontextdaten hinaus kann in der Bibliothek des Zentralarchivs $^{3}$ eine Sammlung von Berichten eingesehen werden, die Informationen zu den Ergebnissen und Kontexten von Landtagswahlen enthalten. Zu den umfangreichen Beständen gehören die Berichte der FGW, die im Auftrag des ZDF entstanden sind sowie die Wahlberichte von infas und Infratest dimap, die im Auftrag der ARD erstellt wurden.

\section{Zugang zu den Daten und Informationen}

Der Daten- und Informationsbestand wird für unterschiedliche Nutzerinteressen über verschiedene Medien zur Verfügung stehen. Die CD-ROM „Landtagswahlen 1962 bis 2004 “ wird den gesamten Datenbestand in einer nutzerfreundlichen Form zugänglich machen und durch den ZA CodebookExplorer die Orientierung in den Landtagswahlstudien erleichtern. Die Ende 2007 verfügbare CD-ROM wird Daten, die für das Projekt von zentraler Bedeutung sind, noch nicht enthalten. Das betrifft einzelne Variablen des integrierten Datensatzes der Umfragedaten sowie die Ereignis- und Makrodaten, die erst nach Abschluss der Projektarbeiten für die wissenschaftliche Öffentlichkeit freigegeben werden.

Die Veröffentlichung der 105 Landtagswahlstudien im Online Study Catalogue des Zentralarchivs (ZACAT) ${ }^{4}$ ermöglicht den weltweiten Zugriff. Mit diesem Angebot, das für nationale und international vergleichende Studienkollektionen Recherchen und Analysen sowie das Herunterladen von Daten und Dokumenten ermöglicht, wird ein breiter Zugang für Forschung und Lehre geschaffen. Das Zentralarchiv arbeitet heute mit Archiven und Instituten aus über 20 Ländern im Council of European Social Science Data Archives (CESSDA) zusammen. Die weiteren Entwicklungen werden die Verknüpfung dieser Daten mit Informationen anderer nationaler und internationaler Portale unterstützen.

3 Spezialbibliothek „Empirische Sozialforschung“ des Zentralarchivs Köln, http://www.gesis.org/ Bibliotheken/ZABIB/.

4 Online Study Catalogue des Zentralarchivs (ZACAT), http://www.gesis.org/Datenservice/zacat. htm. 